\title{
Interview with Dr You Han Bae: Ligand-mediated versus 'passive' targeting approaches in nanoparticle oncology research
}
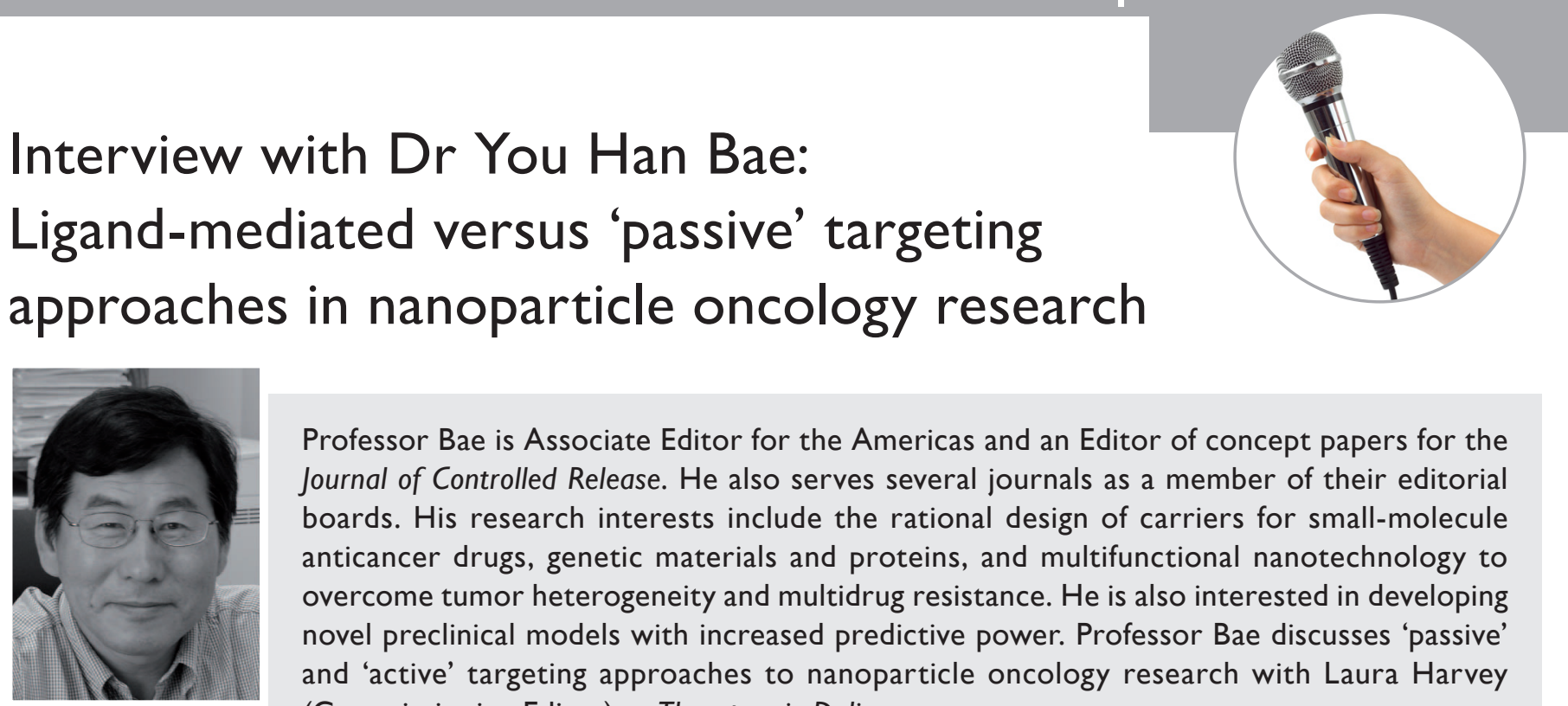

Professor Bae is Associate Editor for the Americas and an Editor of concept papers for the Journal of Controlled Release. He also serves several journals as a member of their editorial boards. His research interests include the rational design of carriers for small-molecule anticancer drugs, genetic materials and proteins, and multifunctional nanotechnology to overcome tumor heterogeneity and multidrug resistance. He is also interested in developing novel preclinical models with increased predictive power. Professor Bae discusses 'passive' and 'active' targeting approaches to nanoparticle oncology research with Laura Harvey (Commissioning Editor) at Therapeutic Delivery.

Q In the nanodelivery field, would you say that terminology such as 'ligand-mediated targeting' is more useful than 'active targeting' given that ligand-targeted particles reach tumor sites by the same mechanism - distribution by the blood - and largely to the same extent as 'passively targeted' particles?

I have been confused with targeting terminology. In fact, scientists in the drug-delivery field have used the terms 'targeting,' 'passive targeting,' 'active targeting' and 'targeted drug delivery' in numerous publications without defining the exact scientific meanings, or with conceptual imagination of missile weapons. Although experienced investigators may know what the terms mean in real-world applications, it often misguides young scientists, journalists and/the general public. 'Targeted drug delivery' implies active mechanisms by which a drug is propelled or transported to its action site. It has been thought that specific interactions between drug carriers and diseased target cells (often malignant cells) endow such 'forced' mechanisms for the carrier to select their target cells. This definitely holds true in in vitro experiments because there is no barrier between the nanocarriers and the cells in cell culture dishes, and all of the carriers can come in close cell contact. Specific interactions only become important after the nanocarrier comes in close contact with its target cell. These interactions are primarily based on the conformational fitting and secondary interactions between a ligand on the nanocarrier and a receptor on the cell surface, which only take place within a $0.1-0.3 \mathrm{~nm}$ range. Such close contact will rarely occur unless nonspecific interactions between the nanocarrier and other proteins are minimized, usually by PEGylation of the carrier surface. When the nanodelivery system is introduced intratumorally or intravenously, the nanocarriers must survive numerous biological and physiological elimination mechanisms before they can reach the target cells. Drug delivery to the tumor site obeys all the physical laws of transport governed by blood flow, convectional transport, Brownian motion, concentration gradient, diffusivity and permeability. The specific interactions have no role in transport until the carriers reach to within $0.3 \mathrm{~nm}$ of the cells. Even the translocation of the nanocarriers from the blood compartment to tumor site by the enhanced permeability and retention (EPR) effect (which is called passive targeting, although it has nothing to do with forced targeting), the transport to individual cells is tremendously limited. For these reasons, I do not favor using the term 'targeting.'

Do you think the 'active/passive targeting' terminology is confusing and causes misunderstandings in defining a specific drug-targeting strategy?

As I mentioned in my response to the first question, the use of 'targeting' is very confusing to me. Targeting (if anybody favors this terminology) is often classified as either passive targeting or active targeting. This classification scheme does not seem to be supported by scientific facts. Cell-specific interactions only occur after close contact with the target cell which, in turn, occurs only after extravasation and transport. Passive targeting is equivalently called the 'EPR

\section{You Han Bae}

Department of Pharmaceutics

\& Pharmaceutical Chemistry,

421 Wakara Way, Suite 315 ,

Salt Lake City, UT 84108, USA

Tel.: +| 8015851518

E-mail:you.bae@utah.edu 


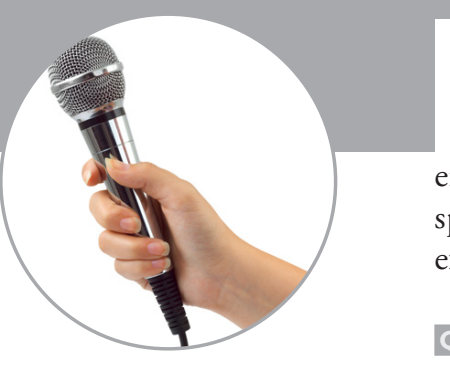

effect' or 'extravation' and assumes free diffusion of the nanocarriers into the tumor interstitial space. This is hardly true for nano-sized carriers in the viscous, interacting and structured extracellular space.

Why do you think there is such variation, from no advantage to modest increase in outcome, from drug delivery via ligand-targeted systems?

A tumor is not a mass of identical cells but rather a complex organ with poorly developed vasculature and numerous cell types, including various stromal cells and cancerous cells, which themselves may vary in phenotype within the tumor. Most biological/physiological systems and events are not truly characterized and predictable like in vitro cell culture systems. The poor predictability becomes more serious in clinical settings than controlled small-animal models. For example, the EPR effect is not reliable except for primary tumors in the liver and kidney. The blood vessel density, distribution and leakiness of solid tumors are not practically known. The transport barriers in the extracellular matrix of a clinical tumor are not measurable before treatment. Tumor heterogeneity in terms of physiology, genetics, epigenetics and microenvironment is not predictable. Moreover, the specificity of 'ligand-receptor interactions' in a real-world environment is not truly defined. A particular receptor on the cell surface is not expressed in a 'yes/no' manner and is not exclusive to target tumor cells, although a fraction of target cancer cells has higher frequency in expression. The tumor mass in a patient may occupy only a small fraction of the whole body weight. Only small fractions of the injected dose of nanocarriers reach the tumor site and this fraction again has very limited distribution within the target tumor. This may explain limited clinical outcomes of nano-delivery systems.

Q What can be/is being done to improve overall results and consistency?

That is a really tough question. Improved and predictable results can be obtained only by modifying the nature of diseased organs in a controllable manner, in a more relevant animal disease model.

Q How do you think the to-date variable outcomes of (proof-of-concept) ligandtargeting studies affects commercial interest and research efforts in ligand-targeting nanotherapies and, conversely, passively targeted systems?

Ligand targeting is conceptually beautiful for in vitro situations but may not be so practical in clinical settings. Various clinical nanotherapies without ligand targeting maximally increased tolerated dose size, altered biodistribution and reduced toxicity profiles. They showed improved clinical outcomes by expanding survival time period in several weeks or several months. It is not truly clear whether the improved outcome is from the effects of nano-sized carriers in a target site or merely from increased doses with reduced toxicity.

Q Ligand-targeted systems are more complex and expensive to manufacture, and more difficult to manufacture in a reproducible manner in particular. In light of this, what do you think of the cost to benefit ratio for the prospect of commercial manufacture of ligand-targeted systems?

This question can be answered only after proving the benefit in clinical systems or equivalent animal models. Considering the medical cost of cancer patients in the last few years before death, if the benefit invcreases years of life, it would be worth developing. However, a developing pharmaceutical company may consider the issue of the market size of a technology before making any decision.

Q How do you see this situation changing? What do you think researchers, clinicians and investors would need to see in terms of proof-of-concept targeting studies for this situation to develop in to the clinic?

No simple technology will be able to cure every tumor. More sophisticated technology that targets a particular disease (tumor) should be developed, which can address the issues of predictable extent of extravasation, intratumoral distribution at a cellular level and tumor heterogeneity, which is relevant to, for example, multidrug resistance, cancer stem-like cells, genetic and epigenetic diversity. 
Q Do you think more attention needs to be paid to the choice of disease that will take advantage of the unique properties of the ligand-targeted systems?

If any tumor expresses unique receptors in over $90 \%$ of the tumor cell population and no other cells express the same receptor, then such a tumor (I do not know such a case exists) would be a good candidate disease to work with ligand targeting. Even then the delivery system should still address other issues.

Q Is it important for scientists working in this field to present their results more objectively, in order to reduce the misrepresentation of the success of targeted nanoplatforms?

There is abundant literature reporting near cures of tumors in immnunocompromised mouse models, while tumors are seldom cured in patients. The results from cell study and small-animal models have always been beautiful with whatever carriers the authors invented. Moreover, many academic technologies are not always reproducible once transferred to industry.

So, are you saying that these results are not misrepresenting the success of targeted nanoplatforms but that readers should always bear in mind the vast differences between in vitro cell cultures/in vivo mouse models to humans?

I assume the investigators do correctly interpret the results from their animal models. These models are in many cases developed by xenografting a particular human cancer cell line or allografting a murine cancer cell line. Although the cell lines adopted contain a certain degree of cell heterogeneity, its extent is significantly less than in clinical tumors. On top of this, animal tumors are different from spontaneous human tumors in many ways: immune environment, tumor microenvironment, the nature of the tumor vasculature, pharmacokinetic/pharmacodynamic behaviors and interactions with host body components. The interpretation of the success of targeted nanoplatforms in animal models may include factors other than targeted nanoplatforms. For instance, the dose schedule in animal models is not equivalent to those applied to human patients. The administration frequency of 1-4 day intervals in animals is significantly higher than a monthly cycle in human patients. I believe we need better animal models, which are significantly more relevant to human diseases, to evaluate targeted drug-delivery systems.

Q Do you think establishing a uniform international system for testing nanoparticulate delivery systems would aid comparison of data from different research groups?

The proposed idea is definitely required, but only after developing more predictable preclinical models that are target-disease specific. Current models with 2D cell culture for screening and immunocompromised mouse models for in vivo efficacy and toxicology often fail to predict organ-specific and systemic toxicity in human patients. After passing current preclinical tests, the success rate of anticancer drugs in clinical trials is below $7-10 \%$.

Should there be a more realistic interpretation of the 'magic bullet concept' - drugs that go straight to their intended cell-structural targets? Should research instead be more realistically aiming for a compound/drug-delivery platform that interacts with its target in a highly specific fashion?

The magic bullet concept is only a hypothetical theory. Even with a system that is highly specific to a target disease, not all cells in a tumor will be affected. Probably only a limited fraction of the cells could be influenced.

Q Should efforts be put towards developing a system that simultaneously aims at multiple targets?

This approach would be a better option to address tumor heterogeneity. However, this can also increase nonspecific interactions with nontarget cells (off-target effect).

Q In what delivery strategies do you think active targeting is really needed?

The so-called 'active targeting' approach would work for liquid cancers such as leukemia, although once a marketed product was withdrawn, because blood cell tumors may have less number of barriers to access and less heterogeneous. But the selection of targeting moiety should be truly 
